Fourth International Symposium on Physics of Fluids (ISPF4)

International Journal of Modern Physics: Conference Series

Vol. 19 (2012) 396-405

(C) World Scientific Publishing Company

DOI: $10.1142 / \mathrm{S} 2010194512008999$

\title{
A NEW EFFICIENT CONTROL METHOD FOR BLENDED WING BODY
}

\author{
WENHUA WU \\ State Key Laboratory of Aerodynamics, Mianyang Sichuan, 621000, China \\ DEHUA CHEN \\ High Speed Institute of CARDC, Mianyang Sichuan, 621000, China \\ NING QIN \\ University of Sheffield, Sheffield S3 7JJ, UK \\ XIN PENG \\ State Key Laboratory of Aerodynamics, Mianyang Sichuan, 621000, China \\ XINWU TANG \\ High Speed Institute of CARDC, Mianyang Sichuan, 621000, China
}

\begin{abstract}
The blended wing body (BWB) is the hottest one of the aerodynamic shapes of next generation airliner because of its' high lift-drag ratio, but there are still some flaws that cut down its aerodynamical performance. One of the most harmful flaws is the low efficiency of elevator and direction rudder, this makes the BWB hard to be controlled. In this paper, we proposed a new control method to solve this problem by morphing wing - that is, to control the BWB only by changing its wing shape but without any rudder. The pitching moments, rolling moments and yawing moments are plotted versus the parameters section and the wing shape in figures and are discussed in the paper. The result shows that the morphing wing can control the moments of BWB more precisely and in wider range. The pitching moments, rolling moments and yawing moments increases or decreases linearly or almost linearly, with the value of the selected parameters. These results show that using morphing wing is an excellent aerodynamic control way for a BWB craft.
\end{abstract}

Keywords: BWB; control; supercritical wing; moment.

\section{Introduction}

To fly farther, faster and to be more fuel efficient is the main design target for not only civil transport but also military cargo plane and some war crafts. In order to achieve these goals, the flying wing and another similar aerodynamic layout called the Blended Wing Body (BWB) are brought back to stage again. The BWB can improve the cruise lift-todrag ratio effectively without the cylindrical fuselage, horizontal tail, and vertical fin etc, parts that don't provide lift but generate drag. The similar aerodynamic configurations have great advantages on aerodynamic efficiency comparing with traditional layouts for its higher lift-to-drag ratio with the same cruising lift coefficient ${ }^{1}$. 
The analysis of drag on typical civil transport at the state of $\mathrm{Ma}=0.8$ and $C_{L}=0.48$ shows that the friction drag, the pressure drag, the induced drag and the parasitic drag respectively take up $51 \%, 19 \%, 27 \%$ and $3 \%$ of the total drag of the whole $\mathrm{craft}^{2}$. It can be seen that the friction drag takes up the biggest part. The friction drag is proportional to the size of the wet area; the civil transport has certain volume which determines its transport capacity for passengers. Thus, the design concept of the BWB is to reduce the wet area and retain the required volume at the same time. The reference paper ${ }^{[1]}$ said that the wet area size of a BWB craft is almost $2 / 3$ of a traditional airliner with the same volume. This is the reason that BWB becomes a hotspot for the new generation of airliners and cargo crafts in recent years.

Another advantage of the BWB and the flying wing layout is their low radar cross section, so that they're less likely to be detected, which makes them fit to be military scout, bomber etc. For example, the stealth bomber B2 has very small radar reflection area with its flying wing layout ${ }^{3}$.

Besides the advantages on cruising efficiency, there're also some disadvantages for the BWB and flying wing, that is its poor directional-lateral and longitudinal controllability and stability ${ }^{3-5}$ caused by follow factors: Generally, the flying wing craft doesn't have the traditional horizontal tail and vertical $\mathrm{fin}^{4-7}$, but its elevator, aileron, rudder blade etc are placed at the trailing edge of the wing, However, the basic function of the traditional vertical fin is to generate yawing and rolling stability, especially yawing stability. The yawing controllability of the craft is also provided by the rudder blade of the vertical fin. Lacking of the vertical fin leads directly to the loss of the directionallateral static stability, and yawing controllability of the craft. Meanwhile, the control surface at the trailing edge is much closer to the gravity center of the BWB comparing with the traditional horizontal tail, which also reduces the lateral and longitudinal controllability and stability.

There is a proven technology to guarantee the balance and controllability for a longitudinal static unstable aircraft, using relaxing longitudinal static stability technology. However, the foundation this technology is to use an artificial stability based on the aircraft's control in the flight control system. Generally a longitudinal static unstable aircraft has a relatively strong pitching controllability. Unfortunately, aircraft without a vertical fin has low longitudinal static stability and pitching controllability at the same time, failing to meet the precondition of the artificial stability ${ }^{6}$.

Researchers at home and abroad did a great deal of work in order to overcome the stability and controllability problems for BWB, presenting a lot of ideas on new control methods. Zuo and $\mathrm{Wang}^{4}$ proposed to use all-moving wingtips to achieve the yaw control. They launched experiments, finding that with the lift remained almost the same, unilateral moving of the all-moving wingtips could provide yawing moment, but associated with large rolling moment and pitching moment as well; the all-moving wingtips' unilateral moving and the side force were coupled slightly; the synchronous moving had similar longitudinal characteristics, but with a larger amplitude. 
Bourdin et al. ${ }^{5}$ put forward an idea to use articulated split wingtips, numerical and experimental results were also provided. It showed that they achieved the longitudinal, lateral, and yawing control by changing the dihedral angles of those split wingtips. Wildschek et al. $^{2}$ raised the idea to use morphing wing tails. They presented the advantages of this method on controlling a flying wing layout aircraft, and designed the mechanism to control the aft wing section's shape.

In this paper, we presented another idea to control flying wing layout aircrafts by wing deformation. We gave different wing deformations respectively to provide pitching, yawing, and rolling moments, and the control torque to generate each deformation. Our research showed that, in aerodynamics, wing deformation is a good control method for flying wing and BWB layout aircrafts. Besides, in this way, there will be no juncture and pits over the surface (one of the main factors of radar reflection and flow separation), the stealth cruise performance and efficiency will also be improved.

\section{Research Method}

In this paper, the research object is a BWB layout aircraft model with large wing spanchord ratio. Numerical method is used to compute the aerodynamic characteristics for the initial shape, and then we redesign the shape by parameterization method, restructured the model, and generated new calculation mesh by mesh deformation. In this paper, numerically, we discussed different wing deformations and the corresponding moments each one generated, the results is used to determine whether certain wing deformation is appropriate for certain control torque, and to select the most efficient one.

\subsection{Research model}

The object of this paper is a BWB layout aircraft model, as shown in Figure 1. The overall dimensions are as follows: $\mathrm{L}$ (wingspan $)=30.0000 \mathrm{~m}, \mathrm{MAC}($ mean aerodynamic chord $)=3.8400 \mathrm{~m}, \mathrm{~S}($ wing area $)=57.5996 \mathrm{~m} 2$. The reference point of moment is at the same position as the midpoint of the chord at the turning point, at the distance of $4.6704 \mathrm{~m}$ from the leading edge of the nose.

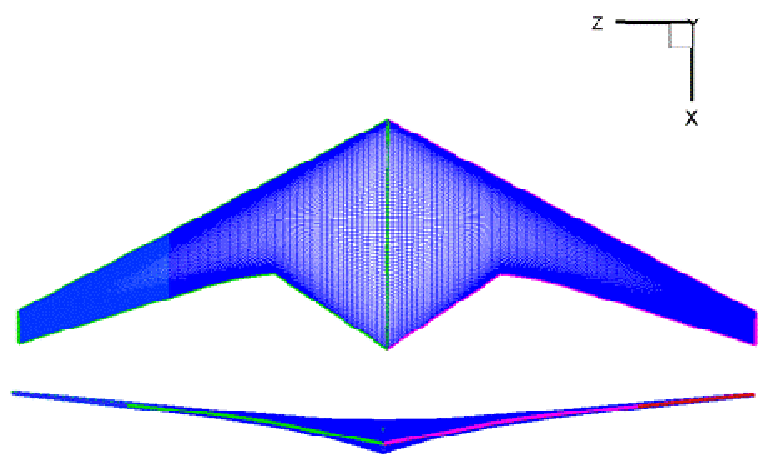

Fig. 1. The BWB layout FLW1 dimension sketch. 


\subsection{Numerical method}

In this paper, a 3D Reynolds averaged Navier-Stokes flow solver that works on structured multiblock grids is developed. The equations are cast in a cell-centred finite-volume form and the convective flux calculation follows Osher's approximate Riemann solver with a MUSCL scheme for higher order accuracy. An implicit method is used for the time discretization $^{8}$. This solver has high precision and good stability, and can control the oscillation of the results effectively.

For an arbitrary control volume $\Omega$, the integral form of the N-S equation is as follows:

$$
\frac{\partial}{\partial t} \iiint_{\Omega} U d V+\iint_{\partial \Omega} F \cdot n d S=0
$$

Among them, $\partial \Omega$ represents the control volume boundary, $F$ represents the matrix of all the $\mathrm{N}-\mathrm{S}$ equations items except time ones.

$$
F=\left[\begin{array}{c}
E_{c}-\frac{1}{\mathrm{Re}} E_{v} \\
F_{c}-\frac{1}{\mathrm{Re}} F_{v} \\
G_{c}-\frac{1}{\mathrm{Re}} G_{v}
\end{array}\right] .
$$

Discretizing the equation above with the finite volume method, the whole calculation domain is divided into many small control volumes. The N-S equations for the control volume of No. $i$ is described as follows:

$$
V_{i} \frac{\partial U_{i}}{\partial t}=-R_{i},
$$

where $U_{i}$ represents the mean value of the state variables of control volume No. $i, R_{i}$ represents the residual of the total flux vector going through the surface of the control volume.

$$
R_{i}=\sum_{\text {faces }} F\left(U_{i}\right) \cdot n S
$$

Time iterative method was applied in this calculation program to solve steady-state problem. The equation for time layer No. $n$ is as follows:

$$
\frac{U^{n+1}-U^{n}}{\Delta t}=-R\left(U^{n}\right) \text {. }
$$

That is:

$$
U^{n+1}=U^{n}-\Delta t R\left(U^{n}\right)
$$

We used the local time step to accelerate convergence. 
The K $\omega$-SST turbulence model is used in the solver. The whole flow field was divided into 11 blocks, with the boundary layer being meshed into a separated block. The thickness for the first layer was 0.000028m; the grid amount was about 1970,000.

\subsection{Shape parameterization and mesh deformation methods}

We need to get the new BWB shape and to generate new mesh for calculation as fast as possible to find out how the control moments changes with to the shape change, and to select the most efficient deformation way giving enough control moment. So, shape parameterization is use to construct new shape quickly. In this paper, we used BezierBernstein method to parameterize the shape, which can present the layout figure more precisely with fewer parameters. Meanwhile, we can make any adjustment to the layout effectively, precisely and meticulously with this method and maintain the smoothness of the shape surface. The main principle of this method is to divide the BWB to a set of wingsections and present each wingsection by two Bezier-Bernstein curves, a few to dozens of control points is used to control the shape of each Bezier-Bernstein curve, the coordinates of these points are the design parameters. The number of curves and points in each curve are determined by the complexity of the layout surface and the optimization requirement on precision. For a general smooth surface, a dozen points will be enough. The traditional Bezier-Bernstein parameterisation for a 2D curve is as follows:

$$
S_{2}(u)=\sum_{k=0}^{N} B_{K, N}(u) P_{k}
$$

In the equation above, $S_{2}(u)=x(u) / y(u), P_{k}=P_{x} / P_{y}$ are the control points of the Bezier curve. In the Bernstein polynomial $B_{K, N}(u)=u^{K}(1-u)^{N-K} \cdot N ! /[K !(N-K) !], u$ represents the reference arc length of the curve, $\mathrm{N}$ represents the number of control points, namely the number of parameters; $P_{x}$ and $P_{y}$ represent the vertical and horizontal coordinates. Take the wing as an example, during the optimization process of a wing, we only need to change the Y coordinate, thus, the design parameter is the Y coordinate $P_{y}$.

We used algebraic method (the transfinite interpolation) for mesh deformation, which can satisfy the basic demand of this study. In this method, the grids on the shape surface are moved to new positions firstly. Then, the change is transferred outwards gradually. During the transfer process, the displacement for each node is calculated in proportion to its initial position, which means the outer boundary of the calculation domain remains unchanged. The new mesh is similar the initial one and mesh topology keeps same, thus the numerical error caused by mesh deformation can be controlled and the results' accuracy can be improved.

\subsection{Basic aerodynamic characteristics of BWB}

The layout of FLW1 is designed for high-altitude flight, with Mach number as 0.8, cruise lift coefficient is 0.44. Its main aerodynamic characteristics are as shown in Figs. 2 and 3 below: 


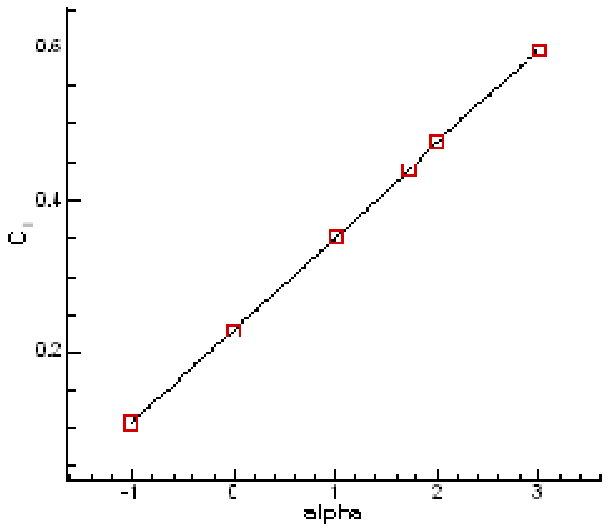

Fig. 2. FLW1's lift characteristics.

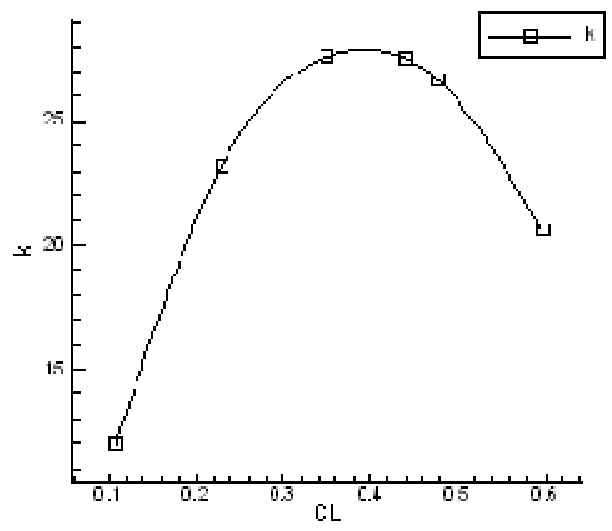

Fig. 3. FLW1's lift-drag characteristics.

It can be seen from the figures that the layout's cruise lift-to-drag ratio reached 27.52, the maximum lift-to-drag ratio reached 27.87 , with a lift coefficient of 0.4 . Within the research range, lift changes linearly with the angle of attack, lift-angle of attack line slope is 0.1208 .

\subsection{Control strategies}

Pitching control strategy:

To get the required pitching control moment by changing the wing section shapes, such as changing camber distribution, thickness, etc. that can change the pressure distribution along the wing chord.

Yawing control strategy:

To generate the yawing control moment by making asymmetric changes to wing section shapes, which makes one side's drag increases, the other one's reduced. In this study, we changed the left wing section shape, keep the right one unchanged.

Rolling control strategy:

To generate the rolling control moment by making asymmetric changes to wing section shapes, thus it generates asymmetric lift. In this study, we only studied changing one wing section shape.

\section{Research Results and Analysis}

We used "superposition method" to parameterize the shape, that is to consider the BezierBernstein parameterization as a variation around this initial shape ${ }^{8}$, then the variation is add to the baseline shape to get the new shape. The advantage of this method is that when all the design parameters are set to be zero, we got an accurate baseline shape.

Three control sections used in the parameterization of FLW1 BWB shape, each section has 16 design parameters, and therefore, there are 48 design parameters in total to describe the whole BWB layout. Its control sections are as shown in Figure 4: the section 
positions are labeled by black line, in each section, there're 8 design parameters on top, and 8 at the bottom, arranged in sequence.

Take the second control section as an example, its control parameters numbered from No.17 to No.32, among which No.17 to No.24 are for the upper surface, No.25 to No.32 for lower surface. These design parameters are arranged from the front to back in sequence, so that the first one in upper surface is No.17, it mainly control the shape of the front part of the upper surface, the last one in upper surface in No.24, mainly control the part near the tailing edge. Figure 5 shows how the shape of the control section changes when the parameters are set respectively as -0.03 and 0.03 . The contour marked by small circles is the initial shape. It can be seen from the figure that, when the parameters are set positive, the back of the wing's lower surface bends upwards, which increases the local camber; on the contrary, decreases the local camber.

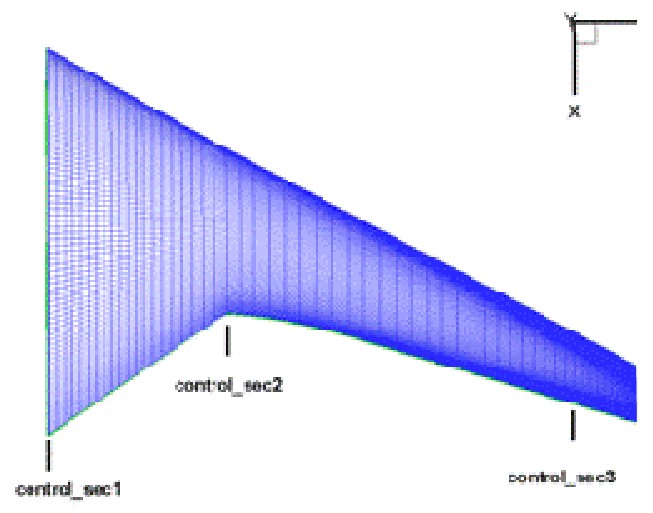

Fig. 4. Control section positions for FLW1 (half model).

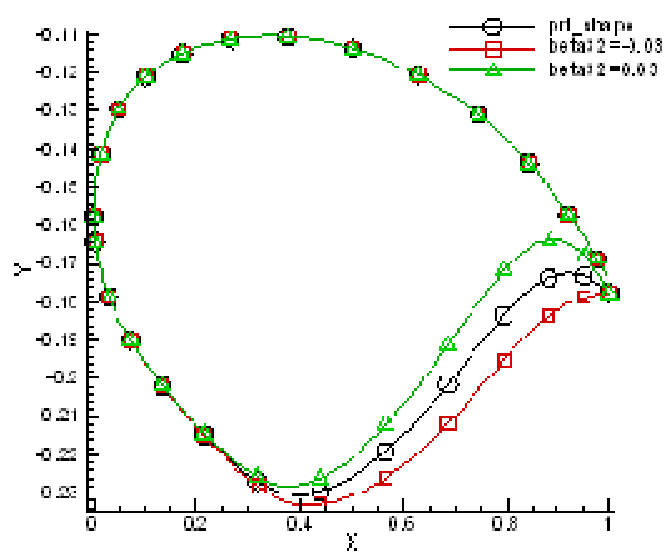

Fig. 5. The deformation of No.2 wing section with its last control parameter in the lower surface.

Figs. 6 to 9 show how the pitching, yawing, rolling moments change with design parameters No.32 and No.16. Specifically, the pitching moment is generated when both 
wings change simultaneously by the same amount; both yawing and rolling moments are generated when the left wing changes with the right one remaining the same.

The pitching moments, rolling moments and yawing moments are plotted versus the parameters No.32 in Figure 6. No.32 is for controlling the back of lower surface of section No.2, while No.16 is used to control the aft part of lower surface of control section No.1. As is shown in Figure 5, when these two parameters are set positive, along with their increases, the back of the wing section's lower surface cave in upwards, accordingly makes the inner part of the wing's lower surface cave in upwards, the lower surface near wing tail edge between control section No.2 and No.3 also sink upwards, and these deformations gradually decrease to zero while approaching control section No.3. As a result, the change that parameter No.32 and No.16 generated is the camber increase at rear of the inner wing part, and the thickness decrease meanwhile. It can be seen from Fig. 6 that pitching moment decreases linearly with the design parameters increasing, namely, the head-down moment increases.

Within the shape deform range researched in this paper, the pitching moment decreases from 0 to -0.08 . It also can be seen from the figure that if these two parameters keep decreasing to below -0.03 to small enough, the rear of the wing's lower surface extrudes downwards more, and the pitching moment may increase linearly to above zero. Further research shows that the rear of the upper surface sinking downwards can generate the same effect. Combining the downwards-extruding of the lower surface and the downwards-sinking of the upper surface, the rising moment will be finally generated until the camber of the wing rear changes to certain value, even the reverse rear camber. Fig. 7 shows how the pitching moment changes with lift when the parameter No.32 changes. It can be seen that there's no obvious change in the slope of the CM-CL curve, which means that the pressure centre doesn't move much, and that the change in the moment is mainly caused by the change of lift. The increase of lift leads to much larger head-down moment because the centre of gravity is in front of the pressure centre. The linear trend of pitching moment curve will make the craft much easier to control.

Fig. 8 shows how the rolling moment changes with design parameters. It can be seen from the figure that, linearly-varying rolling moment can be generated when one side of the wing shape changes. The rolling moment varies in a wide range, from -0.15 to 0.15 in the figure.

Fig. 9 shows how the yawing moment changes with design parameters. We can see from the figure that the yawing moment gradually increases from -0.0038 to 0.0043 with parameter No.32 and No.16, almost linearly, faster when the parameters are bigger.

It can be found in the Ref. 5, which describes the control moments generated by the dihedral variation of split wingtips, that the pitching moment generated by wingtips varies nonlinearly within the discussed range of 0 to 0.025 . The yawing moment also varies nonlinearly, within the range of -0.0025 to 0.0035 . Comparing to the bending split wingtip method, pitching and yawing moments generated by wing deformation method have a wider variation range and better linear characteristics. Therefore, wing deformation is an excellent control method for BWB layout crafts in aerodynamics. 


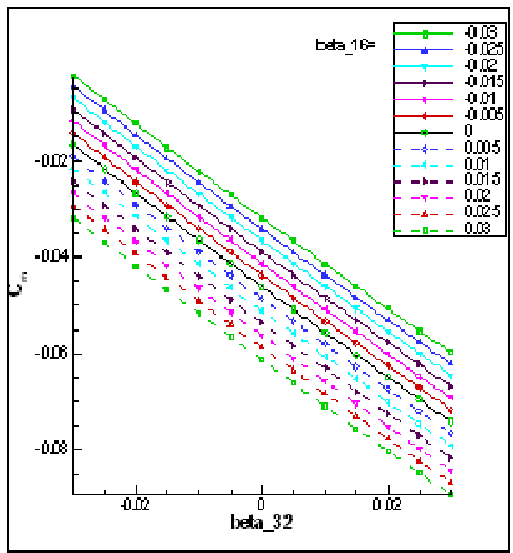

Fig. 6. $C_{m}$ varying with shape deformation.

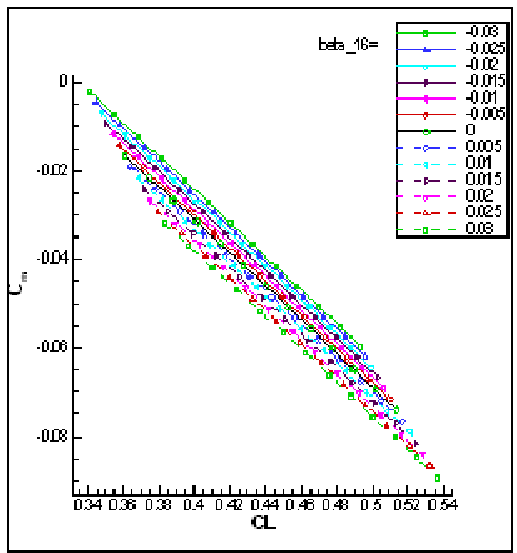

Fig. 7. $C_{m}$ varying with $C_{L}$.

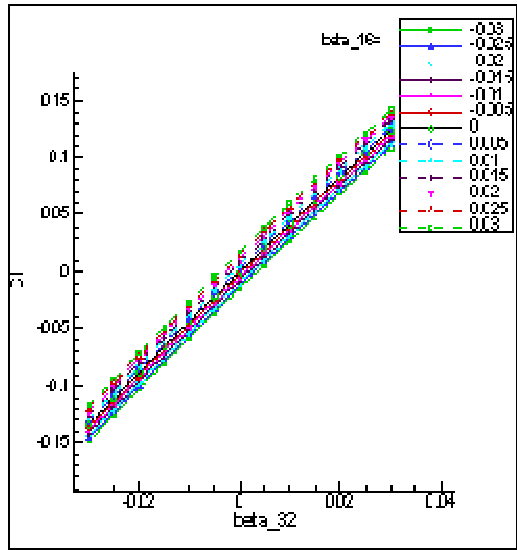

Fig. 8. Rolling moment variation with shape deformation.

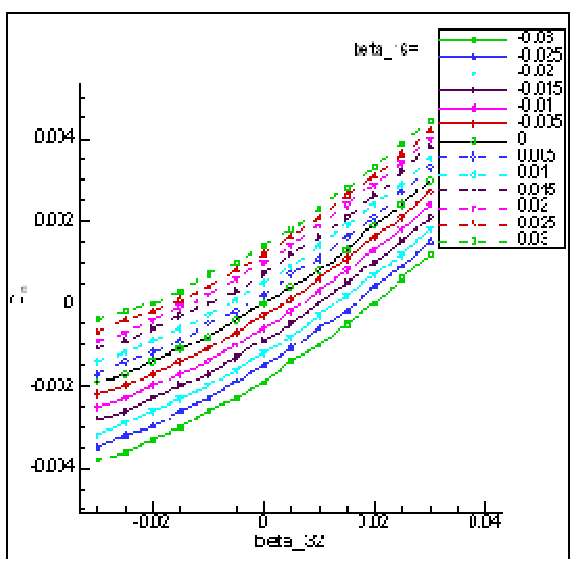

Fig. 9. Yawing moment variation with shape deformation. 


\section{Conclusions}

In this paper, we presented a wing deformation method to achieve the longitudinal, lateral and directional control of a BWB or flying wing layout craft without tail wings. The proper wing deforming patterns for certain control moments is studied.

The pitching, yawing and rolling moments generated in this paper varies linearly with wing deformation, with wide variation ranges and big values, which is profitable for flight control.

From the aerodynamic point of view, wing deformation method has many good features: having linearly changing moments makes it easy to control BWB shapes, keeping wing surface smooth, good for increasing cruising efficiency and Stealth performance. However, further studies are needed to find better deformation strategy that can realize the longitudinal, lateral and orientation control more effectively; how to reduce the interaction among various controls is also a problem to be further discussed.

How to realize the deformation is now the key of the implementation of this technology. So, the deformation mechanism should be developed.

\section{References}

1. N. Qin, A. Vavalle, M. A., and et al., Prog. Aero. Sci. 40, 3212343 (2004).

2. Z. Zhu, X. Wang, Z. Wu and Z. Chen, J. Aero. 29 (2009).

3. A. Wildschek, M. Grünewald, R. Maier and J. Steigenberger, Multi-Functional Morphing Trailing Edge Device for Control of All-Composite, All-Electric Flying Wing Aircraft, in 26th Congress of International Council of the Aeronautical Sciences, 2008.

4. L. Zuo and J. Wang, J. Aero. 28 (2010).

5. P. Bourdin, A. Gatto, and M. I. Friswell, Potential of Articulated Split Wingtips for MorphingBased Control of a Flying Wing, in 25th AIAA Applied Aerodynamics Conference, 2007.

6. Y. Wang, Y. Lu and L. Li, Air. Des. 2005.

7. X. Chai, G. Wang and Z. Wu, Fl. Mech. 27 (2009).

8. A. L. Moigne, A discrete Navier-Stokes Adjoint method for aerodynamic optimization of blended Wing Body configurations, PhD Thesis Cranfield University, 2002 